\title{
Clinicopathological investigation of four cases of desmoplastic small round cell tumor
}

\author{
MEI LI ${ }^{1,2}$, MU-YAN CAI ${ }^{1,2}$, JIA-BIN LU ${ }^{1,2}$, JIN-HUI HOU ${ }^{1,2}$, QIU-LIANG WU ${ }^{1,2}$ and RONG-ZHEN LUO ${ }^{1,2}$ \\ ${ }^{1}$ State Key Laboratory of Oncology in South China; ${ }^{2}$ Department of Pathology, \\ Sun Yat-Sen University Cancer Center, Guangzhou 510060, P.R. China
}

Received February 21, 2012; Accepted May 30, 2012

DOI: $10.3892 / \mathrm{ol} .2012 .750$

\begin{abstract}
The purpose of this study was to perform clinicopathological and immunohistochemical analysis and to investigate the Ewing sarcoma gene (EWS)-Wilms' tumor suppressor gene (WT1) fusion within desmoplastic small round cell tumors (DSRCTs). Histology slides and clinical data were reviewed for four patients with DSRCT. A variety of immunohistochemical staining was performed. Fluorescence in situ hybridization (FISH) was performed to detect the $E W S$-WT1 fusion transcripts resulting from the chromosomal translocation $\mathrm{t}(11 ; 22)(\mathrm{p} 13 ; \mathrm{q} 12)$. The patients consisted of four males aged from 26 to 52 years old (mean, 33.5). In three of these patients, the tumors were situated in the abdominal cavity and the tumor from the other patient was located in the pelvic cavity. The tumors were $8-15 \mathrm{~cm}$ in diameter (mean tumor diameter, 13), solid and gray-white, with an appearance of nodosity or sublobes, and hemorrhage or necrosis was observed. Microscopically, the tumors consisted of small round cell nests of unequal size. Hyperplastic and thick fibrous connective tissue surrounding the neoplastic cell nests was present in all cases. The tumor nuclei were hyperchromatic and contained inconspicuous nucleoli with a high level of karyokinesis. Immunohistochemical staining revealed diffuse and strong staining for $\mathrm{CK}$, vimentin, desmin and CAM5.2 in all cases. Certain cases also expressed WT-1, EMA, NSE, CD56, CD99 and CK5/6. Staining was negative for myogenin, MyoD1, calretinin, CD117, CD34, HMB45 and CEA. EWS-WT1 fusion transcripts were detected in 3 out of 4 cases, but not in any other tumor types studied as controls using paraffin-embedded tissue by FISH. DSRCT is a highly maligant tumor occuring predominantly in the abdominal or pelvic cavity of young males with multiphenotypic differentiation. Basic morphological features, clinical manifestations
\end{abstract}

Correspondence to: Mrs. Rong-Zhen Luo,Department of Pathology, Sun Yat-Sen University Cancer Center, No. 651 Dongfeng Road East, Guangzhou 510060, P.R. China

E-mail: rongzhenluo@163.com

Key words: desmoplastic small round cell tumor, clinicopathological features, immunophenotype, gene fusion and the detection of the EWS-WT1 fusion transcript within the tumor aid the recognition and diagnosis of the tumor.

\section{Introduction}

Desmoplastic small round cell tumor (DSRCT) is a recently recognized clinicopathological entity that is most common in adolescent males and usually affects the abdominal cavity (1). Since first being described in 1989 by Gerald and Rosai (2), DSRCT has been reported in various types of organs, including lung (3), ovary $(4,5)$, pleura (6-8), tunica vaginalis $(9-11)$, soft tissues and bone $(12,13)$, parotid gland (14), central nervous system $(15,16)$ and kidney $(17,18)$. DSRCT is a rare but aggressive malignancy with a poor prognosis and its etiopathogenesis remains unknown. The tumor has a characteristic histology, with extensive stromal tissue surrounding islands of small and undifferentiated cells revealing the desmoplastic appearance (19). DSRCT exhibits a unique immunohistochemical profile, characterized by the coexpression of epithelial (keratin and epithelial membrane antigen), mesenchymal (vimentin) and myogenic (desmin) markers (1). The reciprocal chromosomal translocation $\mathrm{t}(11 ; 22)(\mathrm{p} 13 ; \mathrm{q} 12)$ is specific for DSRCT and this translocation results in the fusion of the Ewing sarcoma gene (EWS) on chromosome 22 with the Wilms' tumor suppressor gene (WT1) on chromosome 11 (20). Undifferentiated tumors often lack the appropriate histopathological features to provide an accurate diagnosis. In the present study, we investigated the expression of molecular markers and EWS-WT1 gene fusion in paraffin-embedded material from four cases of DSRCT.

\section{Patients and methods}

Patients. A total of four male patients from Sun Yat-Sen University Cancer Center (Guangzhou, China) were involved in this study. The patients included three outpatients and a hospital patient. from The patients had provided informed consent for use of tumor specimens. The study was approved by the Institute Research Medical Ethics Committee of Sun Yat-Sen University.

Staining. A review of the clinical data and pathological sections of all cases was performed, representative sections were then selected and immunohistochemically stained. All 
Table I. Pretreatment and working dilutions of primary antibodies used in immunohistochemistry.

\begin{tabular}{llcc}
\hline Antigen detected & Pretreatment & Working dilution & Manufacturer (Cat. No.) \\
\hline CK & HP EDTA & $1: 250$ & Dako (M-3515) \\
Vimentin & - & $1: 400$ & Dako (M-0725) \\
Desmin & HP CB & $1: 150$ & Santa Cruz (sc-70961) \\
CAM5.2 & HP EDTA & Ready for use & ZETA (Z2018) \\
WT-1 & HP EDTA & $1: 80$ & Dako (M-3561) \\
EMA & HP CB & $1: 200$ & Dako (M-0613) \\
NSE & HP EDTA & $1: 150$ & Dako (M-0873) \\
CD56 & HP EDTA & $1: 200$ & Dako (M-7304) \\
CD99 & HP EDTA & $1: 100$ & Dako (IM-3601) \\
CK5/6 & HP EDTA & $1: 250$ & Dako (M-7237) \\
Myogenin & HP EDTA & $1: 100$ & Dako (M-3559) \\
MyoD1 & HP EDTA & $1: 100$ & Dako (M-3512) \\
Calretinin & HP EDTA & $1: 200$ & Dako (M-7245) \\
CD117 & HP EDTA & $1: 150$ & Dako (A-4502) \\
CD34 & HP EDTA & $1: 200$ & Dako (M-7165) \\
HMB45 & HP CB & $1: 80$ & Abcam (ab-787) \\
CEA & HP CB & $1: 300$ & Dako (M-7072) \\
\hline
\end{tabular}

HP CB, boiled with citrate buffer (0.01 mol/l, pH 6.0) under high pressure; HP EDTA, boiled with EDTA (1 mmol/1, pH 8.0) under high pressure; -, no pretreatment.

antibody assays were performed using a standard two-step technique. The pretreatment methods, primary antibodies and their working dilutions used in the present study are listed in Table I. Fluorescence in situ hybridization (FISH) was used to detect the EWS-WT1 fusion. Sections from representative samples were cut into positively charged slices at $4 \mu \mathrm{m}$ thickness. The FISH assays began with deparaffinization of the sections followed by target retrieval (boiled in citrate buffer for $15 \mathrm{~min})$ and pepsin $(4 \mathrm{mg} / \mathrm{ml})$ digestion at $37^{\circ} \mathrm{C}$ for $15 \mathrm{~min}$. For the FISH break apart strategy, the commercial EWSR1 dual color break apart set was used (Vysis Inc., Downers Grove, IL, USA). This combines a 500-kbp Spectrum Orange-labeled probe on the centromeric side of the 7-kbp EWSR1 breakpoint region between exons 7 and 10 of the EWS gene with an 1,100-kbp Spectrum Green-labeled probe localizing on the telomeric side of this breakpoint region. For the FISH fusion approach, a paired set of laboratoryprepared rhodamine-labeled EWS and fluoroisothiocyanate (FITC)-labeled WT-1 were used. The FISH probes were diluted 1:50 in DenHyb buffer (Insitus Laboratories, Albuquerque, NM, USA), hybridization mix (10 $\mu \mathrm{l} / \mathrm{slide})$ was applied to the sections, followed by simultaneous denaturing of the probe and target at $90^{\circ} \mathrm{C}$ for $13 \mathrm{~min}$. Overnight hybridization at $37^{\circ} \mathrm{C}$ occurred in a humidified chamber, followed by post-hybridization washes in $50 \%$ formamide $1 \mathrm{X}$ and $2 \mathrm{X}$ SSC. 4',6-diamidino-2-phenylindole (DAPI; $0.5 \mu \mathrm{l} / \mathrm{ml}$; Insitus Laboratories) was used as a nuclear counterstain. Green and red fluorescent signals were counted in cellular tumor regions with appropriate filters. Sections with sufficient hybridization efficiency (i.e., the majority of nuclei presented a signal) were considered informative, and at least 100 intact and the result was evaluated by two reviewers.

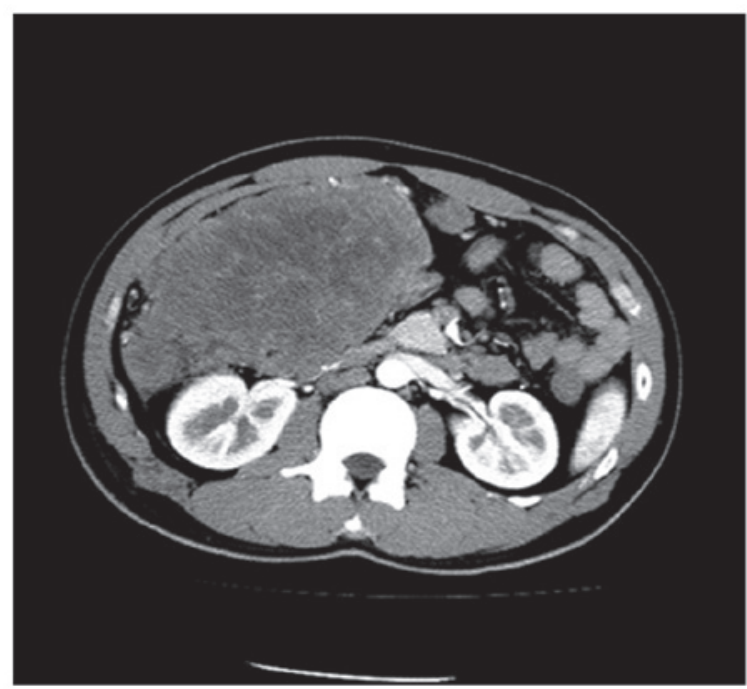

Figure 1. CT scan demonstrates a large, lobular, high density (attenuating) mass from the abdominal cavity of a DSRCT patient. DSRCT, desmoplastic small round cell tumor.

\section{Results}

Clinical data. The eldest patient was 52 and the youngest was 26 years old. B-mode ultrasonography and imageology data showed three tumors situated in the abdominal cavity and one in the pelvic cavity (Fig. 1). Clinical symptoms included abdominal distention, stomach ache and a lump was felt. A few patients had nausea and constipation and one suffered intestinal obstruction. One tumor involved the liver and another involved the intestine. All clinical conditions are listed in Table II. 
Table II. Clinical characteristics and follow-up of the four DSRCT patients.

\begin{tabular}{lcclrr}
\hline Case & Gender & Age (years) & Location of tumor & Size (cm x cm) & Follow-up \\
\hline 1 & Male & 28 & Abdominal cavity & $11.5 \times 8$ & $12 \times 9$ \\
2 & Male & 26 & Abdominal cavity & $13.5 \times 12$ & Deceased \\
3 & Male & 28 & Pelvic cavity & $15 \times 10$ & Deceased \\
4 & Male & 52 & Abdominal cavity & Relapsed
\end{tabular}

DSRCT, desmoplastic small round cell tumor.

Table III. Expression of antibodies used in immunohistochemical analysis of the four DSRCT patients.

\begin{tabular}{lcccc}
\hline Antigen detected & Case 1 & Case 2 & Case 3 & Case 4 \\
\hline CK & + & + & + & + \\
Vimentin & + & + & + & + \\
Desmin & + & + & + & + \\
CAM5.2 & + & + & + & + \\
WT-1 & + & - & - & + \\
EMA & - & + & + & + \\
NSE & - & + & - & + \\
CD56 & - & + & - & + \\
CD99 & + & + & + & - \\
CK5/6 & + & - & + & - \\
Myogenin & - & - & - & - \\
MyoD1 & - & - & - & - \\
Calretinin & - & - & - & - \\
CD117 & - & - & - & - \\
CD34 & - & - & - & - \\
HMB45 & - & - & - & - \\
CEA & - & - & - & - \\
\hline
\end{tabular}

DSRCT, desmoplastic small round cell tumor.

Macroscopic pathological features. The tumors were $8-15 \mathrm{~cm}$ in diameter (mean tumor diameter, $13 \mathrm{~cm}$ ), solid and gray-white, with an appearance of nodosity or sublobes, and hemorrhage or necrosis was observed in three patients.

Microscopic pathological features. The neoplastic cells were arranged compactly and were small, round or orbicular-ovate or short-fusiform with round or orbicular-ovate hyperchromatic nuclei containing inconspicuous nucleoli (Fig. 2A). The karyokinesis was clearly observed and the cytoplasm was diminished. The cells were gathered into nests or clusters with a large amount of hyperplastic and pyknotic fibrous connective tissue among them (Fig. 2B). Necrosis and hemorrhage were observed in certain neoplastic cell nests (Fig. 2C).

Immunohistochemical features. As shown in Table III, the tumor cells of all four DSRCTs expressed CK, vimentin, desmin and CAM5.2 (Fig. 3). Cytoplasm immunoreactivity for desmin presented as punctiform located alongside the nuclei.
Certain DSRCTs also expressed WT-1, EMA, NSE, CD56, CD99 and CK5/6. Staining was negative for myogenin, MyoD1, calretinin, CD117, CD34, HMB45 and CEA (Table III).

FISH. EWS-WT1 fusion transcripts were detected in three of four cases using paraffin-embeded tissue. The EWS-R1 break-apart cocktail revealed separation of signals which is indicative of the translocation involving the EWS locus (Fig. 4).

\section{Discussion}

DSRCT occurs predominantly in adolescents and children with an average age of 21 years, ranging from 3 to 52 years old. The male:female ratio is approximately $5: 1$. A previous study revealed that $95 \%$ of the cases occurred within the abdominal and pelvic cavities, and less than $5 \%$ of cases occur in tissues, including the pleura, adjacent to the testis, brain, liver, lung, mediastinum, paranasal sinuses, ovaries and pancreas (21). Patients with DSRCTs typically present with a large abdominal mass that is widely disseminated at the time of diagnosis. There is often extensive regional spread to the lymph nodes and peritoneal seeding. Metastases may be present in the liver, lung and bone.

Imaging often reveals a large nodular intra-abdominal and/ or pelvic mass, usually located on the omentum or mesentery, with no clear parenchymal organs of primary lesions. The tumor is often a large gray mass, with multiple nodes or lobes, solid and often growing along the plasma membrane. The sections revealed hemorrhage, cystic degeneration or necrosis.

Microscopically, DSRCT has a characteristic appearance of sharply demarcated islands and cords of mitotically active small cells embedded in a dense desmoplastic stroma. The stroma may be associated with hyalinization degeneration and spindle-shaped cells arranged inside as a parallel or fascicular structure which has a fibroblast-like or myofibroblast morphology (22).

Immunohistochemical staining revealed a distinct polyphenotypic pattern consisting of the coexpression of epithelial (cytokeratin, epithelial membrane antigen, CAM5.2), mesenchymal (vimentin, desmin) and neural (neuron-specific enolase) markers. A number of cases have also been reported to express CgA, Syn, CD99, WT-1 and other markers (5). Desmin is of diagnostic value due to the characteristic expression and punctate staining of the nuclei of the tumor cells.

Cytogenetic studies have demonstrated a characteristic reciprocal chromosomal translocation, $\mathrm{t}(11 ; 22)(\mathrm{p} 13 ; \mathrm{q} 12)$. 
A

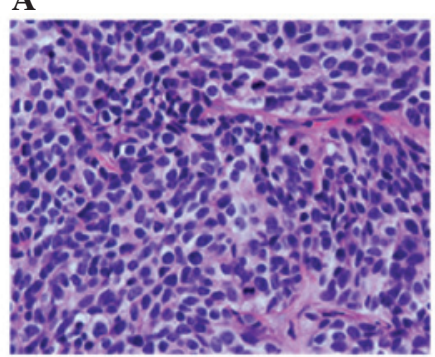

B

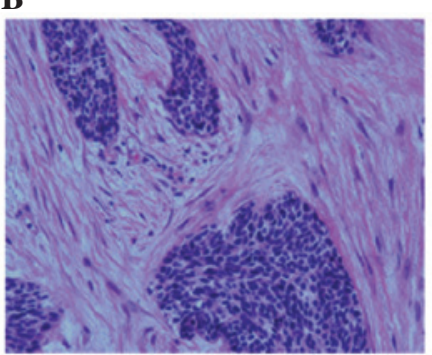

C

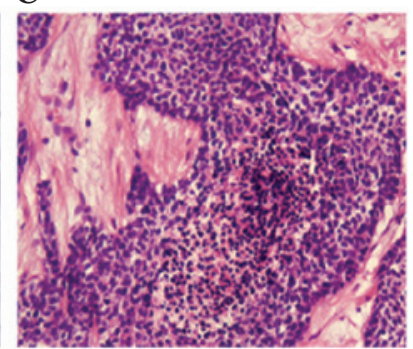

Figure 2. Microscopic features of DSRCT. (a) Neoplastic cells were small, round, arranged compactly with diminished cytoplasm and hyperchromatic nuclei, and karyokinesis was clearly observed (Magnification, $\mathrm{x} 400$ ). (b) Cells gathered as nests or clusters with a high degree of hyperplastic and pyknotic fibrous connective tissue surrounding them (Magnification, x200). (c) Necrosis and hemorrhage was observed in a number of neoplastic cells nests (Magnification $\mathrm{x} 200)$. DSRCT, desmoplastic small round cell tumor.

A

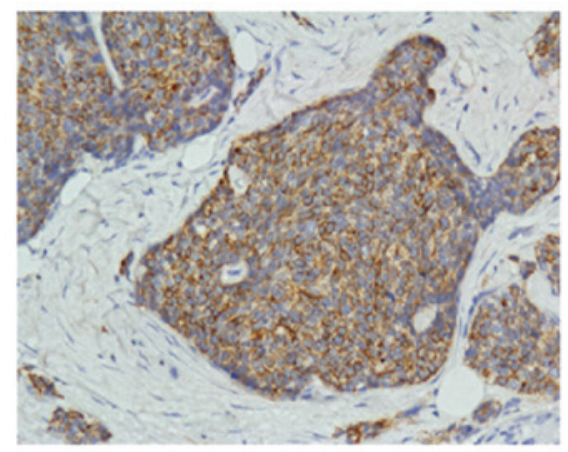

C

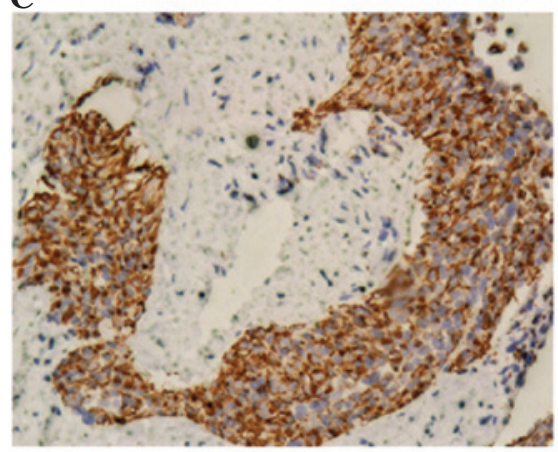

\section{B}

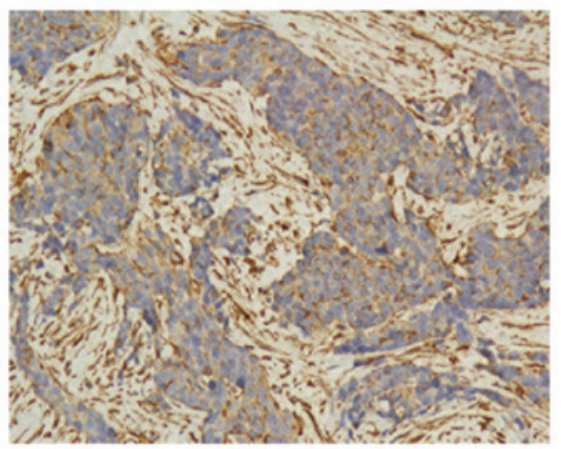

D

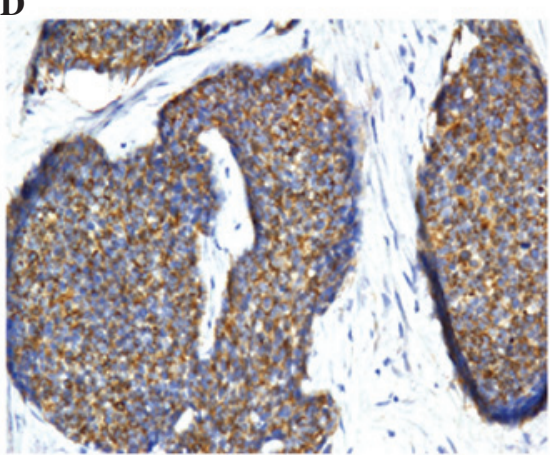

Figure 3. Expression of CK, vimentin, desmin and CAM5.2 in DSRCT. (a) Expression of CK in DSRCT, (b) vimentin, (c) desmin and (d) CAM5.2 in DSRCT (magnification, x200). DSRCT, desmoplastic small round cell tumor.

A

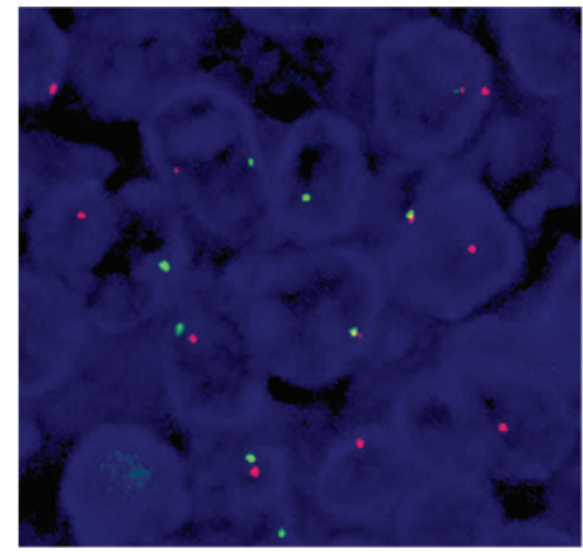

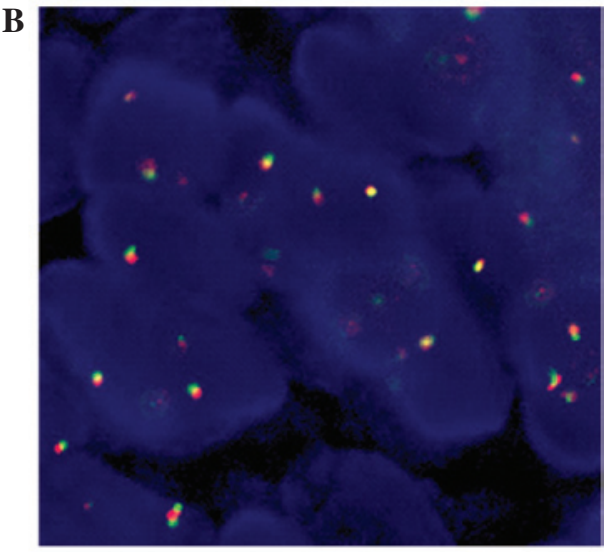

Figure 4. EWS-WT1 fusion transcripts were detected in DSRCT by FISH. (A) EWS-R1 break-apart cocktail showing the separation of signals indicative of translocation involving the EWS locus. (B) Paired locus-specific EWS and WT1 probes show EWS-WT1 (red-green) fusion signals in nuclei of DSRCT. DSRCT, desmoplastic small round cell tumor; FISH, fluorescence in situ hybridization; EWS, Ewing's sarcoma; WT1, Wilms' tumor suppressor gene 1. Magnification, $\mathrm{x} 1,000$. 
The translocation of $\mathrm{t}(11 ; 22)(\mathrm{p} 13 ; \mathrm{q} 12)$ is specific for DSRCT, regardless of its anatomical location $(9,23,24)$. Chimeric EWS-WT1 RNA messages encoded in DSRCTs exhibit significant molecular diversity. EWS combinatorial variability, internal deletion, exon skipping of the coding region sequence and random nucleotide insertions contribute to the heterogeneity of these tumor-specific gene products (25). The chimeric transcript corresponding to the fusion gene product may be detected by the reverse transcription-polymerase chain reaction (RT-PCR) (23), which is highly sensitivitive and specific, and not only suitable for fresh or paraffin-embedded tissue, but may also be applied to fine needle aspiration.

With regard to differential diagnosis, DSRCT must be distinguished from other small round cell tumors, including Ewing's sarcoma/primitive neuroectodermal tumors (PNET), neuroblastoma, rhabdomyosarcoma, malignant mesothelioma, small cell carcinoma, lymphoma, synovial sarcoma and gastrointestinal stromal tumor. Ewing's sarcoma/PNET is typically positive for CD99 and vimentin, but negative for cytokeratins and myogenic markers. The characteristic reciprocal chromosomal translocation in Ewing's sarcoma/ PNET, $\mathrm{t}(11 ; 22)(\mathrm{q} 24 ; \mathrm{q} 12)$, is different from DSRCT, which has a translocation of $\mathrm{t}(11 ; 22)(\mathrm{p} 13 ; \mathrm{q} 12)$. Neuroblastoma occurs mainly in children of less than 5 years old and the stroma is rich in nerve fiber network, often showing ganglion cell differentiation. Tumor cells are negative for CK, desmin and WT1. Rhabdomyosarcoma has often been observed in striated muscle cell differentiation and tumor cells also express MSA, MyoD1 and other myogenic markers, with the exception of desmin. Small cell carcinoma demonstrates immunoreactivity with epithelial markers, including TTF-1 and neuroendocrine markers, but is negative for myogenic markers. Lymphoma often demonstrates a diffuse growth pattern and immunostains show reactivity of lymphoma cells to lymphoid markers but negativity to epithelial and myogenic markers. In contrast to malignant mesothelioma, DSRCT cells are negative for CK5/6, calretinin and other mesothelial markers, and mesothelioma tumor cells are negative for desmin. Approximately $90 \%$ of synovial sarcoma is detected characteristically by SYT-SSX1/2 chromosomal translocation. Similarly, gastrointestinal stromal tumors are detected by the characteristic c-kit gene abnormality.

DSRCT is a highly invasive tumor with a poor prognosis. Rapid progression includes early seeding, as well as hematogenous and lymphatic metastasis. The tumor often metastasizes to the liver, lungs and lymph nodes. Only $29 \%$ of patients survive up to three years and $18 \%$ have a five-year survival rate. No ethnic predisposition or other known risk factors have been identified specifically for the disease (26). To date, early surgical treatment of patients remains the only efficient way to obtain a radical cure. However, most patients are in an advanced stage at the time of treatment and are rarely able to obtain radical resection (27). Several studies suggest a total resection of the mass followed by postoperative multiagent chemotherapy and radiation therapy as the best therapeutic option (26). The ideal therapeutic strategy for treating DSRCT remains unclear due to the rarity of the tumor.

In conclusion, DSRCT is a rare, but highly aggressive malignancy and the prognosis in these patients is extremely poor. Further characterization of the structural and functional attributes of the EWS-WT1 gene fusion may lead to an understanding of its role in tumor development and yield insight into the therapy of DSRCT. A wider availability of specific molecular markers of the tumor would provide auxiliary methods for primary diagnosis and treatment. Prospective genetic therapies focused on developing targeted immunotherapy, moreover, a greater awareness of this disease among pathologists, oncologists, surgeons and biologists, may greatly aid the design of a more effective therapy.

\section{References}

1. Chang F: Desmoplastic small round cell tumors: cytologic, histologic, and immunohistochemical features. Arch Pathol Lab Med 130: 728-732, 2006.

2. Gerald WL and Rosai J: Case 2. Desmoplastic small round cell tumor with divergent differentiation. Pediatr Pathol 9: 177-183, 1989.

3. Syed S, Haque AK, Hawkins HK, Sorensen PH and Cowan DF: Desmoplastic small round cell tumor of the lung. Arch Pathol Lab Med 126: 1226-1228, 2002.

4. Young RH, Eichhorn JH, Dickersin GR and Scully RE: Ovarian involvement by the intra-abdominal desmoplastic small round cell tumor with divergent differentiation: a report of three cases. Hum Pathol 23: 454-464, 1992.

5. Slomovitz BM, Girotra M, Aledo A, Saqi A, Soslow RA, Spigland NA and Caputo TA: Desmoplastic small round cell tumor with primary ovarian involvement: case report and review. Gynecol Oncol 79: 124-128, 2000.

6. Bian Y, Jordan AG, Rupp M, Cohn H, McLaughlin CJ and Miettinen M: Effusion cytology of desmoplastic small round cell tumor of the pleura. A case report. Acta Cytol 37: 77-82, 1993.

7. Parkash V, Gerald WL, Parma A, Miettinen M and Rosai J: Desmoplastic small round cell tumor of the pleura. Am J Surg Pathol 19: 659-665, 1995.

8. Choi JK, van Hoeven K, Brooks JJ and Gupta PK: Desmoplastic small round cell tumor presenting in pleural fluid and accompanied by desmin-positive mesothelial cells. Acta Cytol 39: 377-378, 1995.

9. Rodriguez E, Sreekantaiah C, Gerald W, Reuter VE, Motzer RJ and Chaganti RS: A recurring translocation, $\mathrm{t}(11 ; 22)(\mathrm{p} 13 ; \mathrm{q} 11.2)$, characterizes intra-abdominal desmoplastic small round-cell tumors. Cancer Genet Cytogenet 69: 17-21, 1993.

10. Roganovich J, Bisogno G, Cecchetto G, D'Amore ES and Carli M: Paratesticular desmoplastic small round cell tumor: case report and review of the literature. J Surg Oncol 71: 269-272, 1999.

11. Kawano N, Inayama Y, Nagashima Y, Miyagi Y, Uemura H, Saitoh K, Kubota Y, Hosaka M, Tanaka Y and Nakatani Y: Desmoplastic small round-cell tumor of the paratesticular region: report of an adult case with demonstration of EWS and WT1 gene fusion using paraffin-embedded tissue. Mod Pathol 12: 729-734, 1999 .

12. Antonescu CR, Gerald WL, Magid MS and Ladanyi M: Molecular variants of the EWS-WT1 gene fusion in desmoplastic small round cell tumor. Diagn Mol Pathol 7: 24-28, 1998.

13. Adsay V, Cheng J, Athanasian E, Gerald W and Rosai J: Primary desmoplastic small cell tumor of soft tissues and bone of the hand. Am J Surg Pathol 23: 1408-1413, 1999.

14. Wolf AN, Ladanyi M, Paull G, Blaugrund JE and Westra WH: The expanding clinical spectrum of desmoplastic small roundcell tumor: a report of two cases with molecular confirmation. Hum Pathol 30: 430-435, 1999.

15. Tison V, Cerasoli S, Morigi F, Ladanyi M, Gerald WL, and Rosai J: Intracranial desmoplastic small-cell tumor. Report of a case. Am J Surg Pathol 20: 112-117, 1996.

16. Neder L, Scheithauer BW, Turel KE, Arnesen MA, Ketterling RP, Jin L, Moynihan TJ, Giannini C and Meyer FB: Desmoplastic small round cell tumor of the central nervous system: report of two cases and review of the literature. Virchows Arch 454: 431-439, 2009.

17. Su MC, Jeng YM and Chu YC: Desmoplastic small round cell tumor of the kidney. Am J Surg Pathol 28: 1379-1383, 2004.

18. Egloff AM, Lee EY, Dillon JE and Callahan MJ: Desmoplastic small round cell tumor of the kidney in a pediatric patient: sonographic and multiphase CT findings. AJR Am J Roentgenol 185: 1347-1349, 2005. 
19. Leuschner I, Radig K and Harms D: Desmoplastic small round cell tumor. Semin Diagn Pathol 13: 204-212, 1996.

20. Gerald WL, Rosai J and Ladanyi M: Characterization of the genomic breakpoint and chimeric transcripts in the EWS-WT1 gene fusion of desmoplastic small round cell tumor. Proc Natl Acad Sci USA 92: 1028-1032, 1995.

21. Gerald WL, Miller HK, Battifora H, Miettinen M, Silva EG and Rosai J: Intra-abdominal desmoplastic small round-cell tumor. Report of 19 cases of a distinctive type of high-grade polyphenotypic malignancy affecting young individuals. Am J Surg Pathol 15: 499-513, 1991.

22. Ferlicot S, Coué O, Gilbert E, Beuzeboc P, Servois V, Klijanienko J, Delattre O and Vielh P: Intraabdominal desmoplastic small round cell tumor: report of a case with fine needle aspiration, cytologic diagnosis and molecular confirmation. Acta Cytol 45: 617-621, 2001.

23. Ladanyi M and Gerald W: Fusion of the EWS and WT1 genes in the desmoplastic small round cell tumor. Cancer Res 54 2837-2840, 1994.
24. Sawyer, JR, Tryka AF, and Lewis JM: A novel reciprocal chromosome translocation $\mathrm{t}(11 ; 22)(\mathrm{p} 13 ; \mathrm{q} 12)$ in an intraabdominal desmoplastic small round-cell tumor. Am J Surg Pathol 16: 411-416, 1992.

25. Liu J, Nau MM, Yeh JC, Allegra CJ, Chu E and Wright JJ: Molecular heterogeneity and function of EWS-WT1 fusion transcripts in desmoplastic small round cell tumors. Clin Cancer Res Sep 6: 3522-3529, 2000

26. Koniari K, Mahera H, Nikolaou M, Chatzis O, Glezakou O, Magiasis V and Kiratzis G: Intraabdominal desmoplastic small round cell tumor: Report of a case and literature review. Int J Surg Case Rep 2: 293-296, 2011.

27. Kushner BH,LaQuaglia MP, Wollner N, Meyers PA,Lindsley KL, Ghavimi F, Merchant TE, Boulad F, Cheung NK, Bonilla MA, et al: Desmoplastic small round-cell tumor: prolonged progression-free survival with aggressive multimodality therapy. J Clin Oncol 14: 1526-1531, 1996. 